\title{
Die Wiederkehr der Zünfte? Netzwerkbeziehungen aus historischer Perspektive $^{1}$
}

\section{Einleitung}

Nach Marc Amstutz und Gunther Teubner liegt eine der Herausforderungen, die das Entstehen der Netzwerke in den letzten Jahrzehnten des 20. Jahrhunderts an uns stellt, in ihrer Inkompatibilität mit rechtlichen Begriffen wie Vertrag und Gesellschaft, was im Grundsatz der seit der industriellen Revolution vorherrschenden Unterteilung in Markt und Unternehmen entspricht. ${ }^{2}$ Der folgende Beitrag stellt sich der Herausforderung, den Platz der Netzwerke im langen Prozess der Industrialisierung genauer zu bestimmen und ihn auf den Rechtsbegriff des Unternehmens zu beziehen. Manuel Castells ${ }^{3}$ betrachtet Netzwerke als Erscheinung der »postindustriellen Gesellschaft« des späten zwanzigsten Jahrhunderts. Doch wie würden wir das Phänomen Netzwerk interpretieren, wenn wir statt dessen feststellten, dass Netzwerke auch schon vor dem Entstehen des modernen Industrieunternehmens bestanden?

Vor der industriellen Revolution war die Zunft die vorherrschende Form der wirtschaftlichen Organisation in Westeuropa und Nordamerika. Sie besaß viele der Eigenschaften, die man heute Netzwerken zuschreibt. Zünfte waren weder Unternehmen noch Märkte, sondern lose Zusammenschlüsse unabhängiger Hersteller mit starker lokaler oder regionaler Prägung. Sie kombinierten Kooperation und Wettbewerb und die gesamte Branche teilte sich die Vorteile der Innovation. Ihre Wertorientierung waren enge Zusammenarbeit, Solidarität der Hersteller und die Verteidigung des Handwerks als >geistiges Gemeinschaftsgut $<$. Doch eben diese Werte konnten auch als Kollusion, wettbewerbswidrige Absprachen und Monopolisierungstendenzen interpretiert werden. Für die englischen Richter im 17. und 18. Jahrhundert stellten Zünfte buchstäblich eine »Verschwörung « gegen die Verbraucher und die Interessen der Gesamtgesellschaft dar. In der Zeit, als das Common Law die Grenzen der strafrechtlichen und zivilrechtlichen Haftung für unzulässige »Verbindungen « neu festlegte und die Lehre von der »Handelsbeschränkung « entwickelte, wurden die Zünfte auch von der Gesetzgebung der französischen Revolution und den post-revolutionären Gesetzbüchern des europäischen Kontinents verurteilt. Der Untergang der Zünfte ebnete zwar den Weg für das Entstehen integrierter Wirtschaftsunternehmen, doch dieser Übergang war bei weitem nicht nahtlos. Einige Aspekte des Zunftwesens lebten auch unter den Bedin-

1 Für ihre Anregungen danke ich Ana Lourenço, Steve Pratten, Mathias Siems und Frank Wilkinson, sowie den Teilnehmern der Konferenz in Freiburg und insbesondere Poul Kjaer. Mein Dank gilt auch Josefine M. Jaber und Brigitte Mauel für die Übersetzung meines Textes in die deutsche Sprache.

2 Marc Amstutz und Gunther Teubner (2006) »Editorial«, in diesem Heft.

3 Manuel Castells (1999) The Information Age: Economy, Society and Culture. The Rise of the Network Society (Volume 1), Oxford: Blackwell. 
gungen des industriellen Kapitalismus weiter, wenn auch in Nischen und Segmenten, die ständig von Übergriffen bedroht waren.

Zunft und Netzwerk sind keine synonymen Begriffe; doch Zünfte sind eine besondere Art von Netzwerk, denn sie basieren auf >lateralen bzw. horizontalen Austauschmustern, voneinander abhängigen Ressourcenströmen und wechselseitiger Kommunikation ${ }^{4}$. Aus historischer Perspektive ist nun das Schicksal der Zünfte besonders lehrreich. Die These heißt: Wenn die Netzwerke des (post)-industriellen Kapitalismus nicht wie einst ihre Vorgänger an den Rand des wirtschaftlichen Geschehens gedrängt werden sollen, ist es notwendig, sie, wenn auch nicht unbedingt durch eine umfassende Rechtsdogmatik, dann aber doch wenigstens durch eine Reihe von Rechtskonzepten und -verfahren zu stützen und zu schützen. In dem Maße, wie das Recht die Bildung von Unternehmen und Märkten zulässt, müssen auch Netzwerkbeziehungen rechtlich abgesichert werden.

Mit diesem Ziel vor Augen ist der Beitrag zunächst bemüht, Netzwerke im wirtschaftlichen und rechtlichen Kontext zu definieren und die Beziehung zwischen Netzwerken, Märkten und Unternehmen zu klären. Es folgt eine historische Analyse der rechtlichen Veränderungen, die die Zünfte zunächst unterstützten, später aber ihren Untergang begleiteten. Im Anschluss daran soll die Rolle beleuchtet werden, die Wettbewerbsrecht und -politik bei der Neudefinition der Organisationsgrenzen in einem prototypischen >post-industriellen $<$ Sektor, der Kulturindustrie, spielten. Am Ende stehen rechtspolitische Folgerungen.

\section{$>$ Markt<, >Unternehmen $<$ und >Netzwerk $<$ als wirtschaftliche und rechtliche Konzepte}

Eine Schlüsselfrage ist, ob sich Märkte, Unternehmen und Netzwerke gegenseitig ausschließen. Die Transaktionskostenökonomik in der Tradition von R.H. Coase ${ }^{5}$ und Oliver Williamson ${ }^{6}$ stellt Unternehmen und Märkte als alternative Formen wirtschaftlicher Organisation dar. Es wurde jedoch nie überzeugend nachgewiesen, dass Netzwerke eine spezifische Organisationsform in diesem Sinne bilden. Jeffrey Bradach und Robert Eccles, ${ }^{7}$ die in >Preis, Autorität und Vertrauen $<$ mögliche alternative Mechanismen zur Koordinierung von Märkten, Unternehmen oder Netzwerken sahen, haben letztendlich zu akzeptieren, dass diese nur Idealtypen sind, die sich zwar für Denkmodelle sehr gut eignen, jedoch nicht die Realität industrieller Organisation widerspiegeln. Als Kontrollmechanismen können sie, obwohl >eigenständigく, in der Praxis auch >überlappen, eingebettet und miteinander verflochten sein, nebeneinander stehen und ineinander passen $<$. Die Annahme, dass sie sich gegenseitig ausschließen,

4 Walter Powell (1990) »Neither market nor hierarchy: network forms of organisation«, 12 Research in Organizational Behavior, 295-336, Wiederabdruck in: Grahame Thompson, Jennifer Frances, Rosalind Levacic und Jeremy Mitchell (Hg.) (1991) Markets, Hierarchies and Networks: The Coordination of Social Life, London: Sage, 265-275, 265.

5 Ronald Coase (1937) »The nature of the firm«, 4 Economica (NS), 386-405.

6 Oliver Williamson (1986) The Economic Institutions of Capitalism, New York: Free Press.

7 Jeffrey Bradach und Robert Eccles (1989) »Price, authority and trust: from ideal types to plural forms«, 15 Annual Review of Sociology, 97-118. 
folgern diese Autoren, >trägt eher zu einem schlechteren als zu einem besseren Verständnis bei $<{ }^{8}$

Ebenso war Walter Powell darum bemüht, >eine Reihe von stimmigen Faktoren, die es sinnvoll machen, über Netzwerke als eine bestimmte Form der Koordinierung wirtschaftlicher Aktivitäten zu sprechen $<$, herauszuarbeiten. ${ }^{9}$ In seiner Abhandlung sind Netzwerke >sozialerくals Märkte, das heißt >abhängiger von Beziehungen, gegenseitigen Interessen und Reputationen $<{ }^{10}$, dabei jedoch $>$ weniger geleitet von einer formalen Autoritätsstruktur ${ }^{11}$ als Hierarchien. Im Unterschied zu Märkten verfügen Netzwerke über Mechanismen des sozialen Lernens und der Informationsübertragung, während sie im Gegensatz zu Hierarchien von >wechselseitiger Orientierung « und Reziprozitätsregeln abhängig sind. ${ }^{12}$ Schwierig an dieser Analyse ist, dass die Merkmale, die Powell als netzwerkspezifisch beschreibt, in überzeugender Weise auch auf die beiden anderen Formen zutreffen können. Wie F.A. Hayek zeigte, ${ }^{13}$ dienen Märkte dazu, Wissen zu mobilisieren und zu kodieren. Hayek mag in seiner Darstellung vielleicht übertreiben, wenn es um das Maß geht, in dem dies ein notwendiges oder universelles Merkmal marktbasierter Aktivität ist, doch ohne Frage ist es in vielen Marktbereichen präsent. Umgekehrt legen viele Unternehmen, wenn auch nicht alle, auf ein hohes Maß an Vertrauen und Zusammenarbeit zwischen Arbeitern und Unternehmensführung Wert, ohne dass sie dabei den Charakter einer auf Hierarchie aufgebauten Organisation verlieren.

Trotzdem spricht einiges dafür, dass Netzwerke typische Merkmale besitzen. Das Problem der bisherigen Studien liegt darin, dass sie von der Coaseschen Annahme ausgehen, dass Unternehmen und Märkte Alternativen sind. Das Unternehmen ersetzt den Markt, wenn die Organisationskosten für Transaktionen durch externen Austausch steigen. Dies ist im Grunde eine eher statische Betrachtungsweise, die bestenfalls, wie Coase sagt, einem >moving equilibrium< nahe käme. ${ }^{14}$ Aus evolutionärer oder historischer Sicht dürfen aber Unternehmen und Markt nicht als Alternativen gesehen, sondern müssen als sich gegenseitig ergänzende Strukturen betrachtet werden. Das Entstehen des vertikal integrierten Unternehmens und der damit verbundenen Organisationstechniken der Massenproduktion ging Hand in Hand mit dem Aufkommen von Märkten, die auf dem Massenkonsum standardisierter Waren basierten. In der Sprache der modernen Systemtheorie könnte man sagen, dass sie >koevolvierten $<{ }^{15}$

8 Bradach und Eccles (Fn. 4) 116.

9 Powell (Fn. 4) 268.

10 Powell (Fn. 4) 268.

11 Powell (Fn. 4) 268.

12 Powell (Fn. 4) 272.

13 Siehe vor allem Friedrich Hayek (1982) Law, Legislation and Liberty. A New Statement of the Liberal Principles of Justice and Political Economy, London: Routledge.

14 Coase (Fn. 5).

$15 \mathrm{Zu}$ Ko-evolution und Systemtheorie im Hinblick auf die Entwicklung von Rechtsformen, siehe allgemein Gunther Teubner (1989) Recht als autopoietisches System, Frankfurt: Suhrkamp, 61ff.; Marie Theres Fögen (2002) »Rechtsgeschichte - Geschichte der Evolution eines sozialen Systems«, 1 Rechtsgeschichte, 14-19; englische Fassung abrufbar unter: http://www.mpier.uni-frankfurt.de/Forschung/Mitarbeiter_Forschung/foegen-legalhistory.htm; Marc Amstutz (2001) Evolutorisches Wirtschaftsrecht, Baden-Baden: Nomos. 
Diesen Prozess beschrieb J. R. Commons 1909 in einer im Quarterly Journal of Economics erschienenen (heute wäre das wahrscheinlich nicht mehr möglich) klassischen und in letzter Zeit sträflich vernachlässigten Abhandlung über die industrielle Entwicklung sehr genau - ohne dabei diese spezielle Terminologie zu verwenden. ${ }^{16} \mathrm{Com}$ mons äußerst detaillierte >mikro-institutionelle< Analyse verfolgt die Entwicklung der Schuhindustrie in Amerika von den Zünften zur Zeit der frühen Kolonialzeit bis hin zur Fabrikarbeit im Zeitalter der Massenproduktion. Wie Commons erkannte, repräsentierte die >ursprüngliche ${ }^{17}$ Zunft >gleichermaßen die später getrennten Klassen der Kaufleute, Handwerksmeister und Gesellen $<.^{18}$ Die hauptsächliche Aufgabe der Zunft bestand darin >schlechte Ware < auszuschließen. ${ }^{19}$ In der Praxis lief dies oft darauf hinaus, dass das Vertragsrisiko auf den Verbraucher verlagert wurde. Die spätere Zersplitterung der Zünfte war gemäß Commons nicht nur das Ergebnis von technischen oder organisatorischen Veränderungen im Produktionsablauf, obwohl diese auch eine Rolle spielten, sondern vor allem eine Folge der `Erweiterung < bisheriger oder der Erschließung neuer Absatzmärkte. ${ }^{20}$ Dies führte zu einer Aufspaltung des Handels in Groß- und Einzelhandel und damit zu einer entscheidenden Verlagerung der wirtschaftlichen Macht vom Hersteller auf den Verbraucher: Es kommt somit dazu, >dass der ständig wachsende Markt von der Auftragsfertigung, über den Einzelhandel und den Großhandel hin zum Großhandelsspekulationsgeschäft, den Gesellen mehr und mehr von seinem Markt verdrängt, die Aufmerksamkeit mehr auf den Preis als auf die Qualität gelenkt und der Gewinn aus dem jeweiligen Geschäft vom Gesellen auf den Verbraucher und Zwischenhändler übertragen wird ${ }^{21}$ Dieser Wandel vollzog sich ohne große Änderungen des Produktionsprozesses und vor der Gründung der Fabriken, die nach Commons lediglich eine Begleiterscheinung auf dem Weg zur letzten Stufe der Marktexpansion waren, die er im Zeitalter der Massenproduktion als $>$ die Welt< umspannend sah. ${ }^{22}$

Die vertikale Integration mit der Ausdehnung von Märkten auf globaler Ebene gleichzusetzen, widerspricht zwar der erst in der jüngsten Vergangenheit entstandenen gedanklichen Verbindung der Globalisierung mit einer >flexiblen<, post-industriellen und im wesentlichen zersplitterten Wirtschaftsstruktur. ${ }^{23}$ Doch was Commons beschrieb, war ein Prozess, den zeitgenössische Kommentatoren als eine Entmachtung der Arbeitskräfte verstanden. In den ersten Jahren des 20. Jahrhunderts wehrten sich gleichermaßen Handwerker-Gewerkschaften und kleine Hersteller gegen die vertikale Integration, denn sie waren sich bewusst, dass sie eine Bedrohung ihrer Unabhängig-

16 John Commons (1909) »American shoemakers, 1648-1895: a sketch of industrial evolution«, 24 Quarterly Journal of Economics, 39-84.

17 Commons (Fn. 16) 43.

18 Commons (Fn. 16) 42-43.

19 Commons (Fn. 16) 43.

20 Commons (Fn. 16) 50.

21 Commons (Fn. 16) 67.

22 Commons (Fn. 16) 84 (»Appendix II. Shoemakers - Industrial Stages, Causes, and Organizations «) Spalte 1 (»Extent of Market«) letzte Zeile.

23 Castells (Fn. 3). 
keit darstellte. ${ }^{24}$ Die Rechtskategorie des >Arbeitsvertrages< verbreitete sich damals zum größten Teil in Folge des Wunsches der Arbeitgeber nach einer einheitlichen Rechtsstellung, welche die >Unterordnung< aller Lohn und Gehalt beziehender Arbeiter unter die Autorität der Unternehmensleitung nach sich zog. ${ }^{25}$ Seitdem haben wir uns daran gewöhnt, das große vertikal integrierte Unternehmen und den >festen $<$ bzw. unbefristeten Arbeitsvertrag als Institutionen zum Schutze der von einer Zersplitterung des Unternehmens bedrohten Interessen der Arbeitnehmer zu betrachten. Doch Unternehmen und Arbeitsverträge waren dies zu Beginn nicht und Commons, der seine Abhandlung 1909 schrieb, hätte sie auch so nicht gesehen.

Commons hebt die gleichzeitige Entwicklung von Unternehmen und Märkten als Folge der Wettbewerbsausweitung hervor, weist aber gleichzeitig auch auf die Ausgrenzung der Netzwerkformen hin. Da Unternehmen und Markt sich gegenseitig ergänzten, drängte ihr gemeinsamer Aufstieg die Netzwerke an den Rand. Dies geschah einerseits in Folge der Eingliederung von unabhängigen Einheiten in das Unternehmen und andererseits durch das Verschwinden der auf die Kunden zugeschnittenen Produktion durch die Massenkonsummärkte. Netzwerke überlebten, jedoch nur in isolierten Zusammenhängen, Segmenten und Nischen, die um ihr Überleben kämpfen mussten, sobald sie dem direkten Wettbewerb mit integrierten Industrieformen ausgesetzt wurden.

Zusammenfassend lässt sich daraus folgern: Netzwerke sind in der Tat eine besondere Form der Organisation, doch bieten sie nicht eine Alternative zum Unternehmen einerseits und zum Markt andererseits, sondern eine Alternative zur Verbindung von vertikal integrierten Unternehmen und Massenkonsummärkten. Durch den sich entwickelnden industriellen Kapitalismus wurden Netzwerke zum größten Teil von der Verbindung Unternehmen-Markt ausgegrenzt. Sie sind jedoch niemals ganz verschwunden. In manchen Bereichen florierten sie sogar, doch dies blieb die Ausnahme.

Welche Rolle spielte der rechtlich-institutionelle Rahmen in diesem Prozess? Im nächsten Abschnitt wird untersucht, welche Rolle die Veränderungen im englischen Recht spielten, die den Übergang zum industriellen Kapitalismus begleiteten.

Die rechtliche Grundlage der Zunftbildung und der Übergang zum industriellen Kapitalismus in England

Die Bildung von Zünften im frühen modernen England war kein bloßes Relikt des Mittelalters. Historische Forschungen haben erst kürzlich die Rolle der Zünfte neu be-

24 Siehe Sanford Jacoby (1985) Employing Bureaucracy: Managers, Unions, and the Transformation of Work in American Industry 1900-1945, New York: Columbia University Press; David Marsden (1999) A Theory of Employment Systems: Micro-Foundations of Societal Diversity, Oxford: Oxford University Press; Peter Cappelli (2002) »Market-mediated Employment: The Historical Context«, in: Margeret Blair und Thomas Kochan (Hg.) The New Relationship. Human Capital in the American Corporation, Washington DC: Brookings Institution, 66-90.

25 Simon Deakin und Frank Wilkinson (2005) The Law of the Labour Market: Industrialisation, Employment, and Legal Evolution, Oxford: Oxford University Press. 
wertet. Es wurde daran erinnert, dass sie bei weitem nicht das Fortschrittshemmnis waren, wie sie die damalige politische Ökonomie dargestellt hat. Es gibt Anhaltspunkte dafür, dass die Zünfte die >proto-industriellen< Produktionsformen maßgeblich unterstützten, die auf einer Reihe komplexer vertraglicher Beziehungen zwischen Händlern, Großhändlern und Herstellern basierten und welche die Gesamtwirtschaft vor dem Aufkommen der Fabrik charakterisierten. ${ }^{26}$ Zur damaligen Zeit wurden die Zünfte von einer sorgfältig ausgearbeiteten und umfassenden Rechtsstruktur unterstützt. ${ }^{27}$ Selbst noch Mitte des 18. Jahrhunderts war es notwendig, eine 7-jährige Lehrzeit zu absolvieren, um Zugang zu gewissen Handwerksberufen zu erlangen. Lehrlinge erhielten ihren Lohn nur zum Teil in bar - ein Teil ihres Lohnes bestand aus Sachleistungen, sowie Kost und Logis im Haushalt des Lehrmeisters. Als Gesellen bezeichnete man jene, die ihre Lehrzeit in dem entsprechenden Handwerk abgeschlossen hatten. Sie wurden im allgemeinen auf täglicher Basis entlohnt, obwohl sie in der Regel für längere Zeit angestellt wurden. Ein Geselle wurde nicht einfach in den Kreis der Meister aufgenommen, wenn er die entsprechenden finanziellen Mittel und Qualifikationen vorweisen konnte, die er als unabhängiger Gewerbetreibender dafür benötigte. Gemäß den Regeln der Zunft war nämlich die Anzahl der Meister streng begrenzt. Damals hatten die Begriffe >Meister $<$ und >Arbeitgeber $<$ nicht die gleiche Bedeutung. ${ }^{28}>$ Meister $<$ war jemand, der auf Grund seines beruflichen Fachwissens und in vielen Fällen auf Grund seiner Stellung als Freier der entsprechenden Zunft oder Stadtvereinigung das Recht hatte, die bei ihm angestellten Lehrlinge und Gesellen anzuleiten. Der Meister konnte dann wiederum als unabhängiger Unternehmer, auf regelmäßiger oder unregelmäßiger Basis, von dritten Parteien wie Händlern oder reichen Kunden >angestellt< werden.

Das Handwerkersystem unterscheidet sich von den späteren kapitalistischen Beschäftigungsformen durch die Beibehaltung der Kontrolle über Art und Schnelligkeit der Arbeit durch das >Handwerk <, mit anderen Worten, durch die Gesamtheit der Hersteller, die den Regeln der Zunft unterworfen waren. Das Verhältnis des Meisters zu seinen Lieferanten und Kunden entsprach, selbst wenn es regelmäßiger und stabiler Art war, nie dem eines Angestellten (so wie man diesen Begriff später verstand). Ein Geselle, der auf täglicher Basis oder pro Stück entlohnt wurde, konnte nur in seinem erlernten Beruf tätig werden, doch zugleich war er durch die Beschränkung der Lehrlingszahl und durch die allgemeinen Kontrollen vor der Aufnahme in das Handwerk vor dem Wettbewerb durch Niedriglöhne geschützt. Auf diese Art und Weise war das >Handwerker-Entlohnungs-System < eine Beziehung, in der ein Geselle >mit seinem Meister und nicht für ihn arbeitete und in Zeiten, in denen es wenig Arbeit gab, wurde das Arbeitsverhältnis wahrscheinlich so lange aufrecht erhalten, wie es dem Meister möglich war $<$. Die Regeln der Zunft boten dem Meister wiederum eine >geschützte

26 Siehe Martin Daunton (1995) Progress and Poverty: An Economic and Social History of Britain 1700-1850, Oxford: Oxford University Press, 155-158.

27 Siehe Deakin und Wilkinson (Fn. 25) für umfassendere Informationen hinsichtlich der in dem Abschnitt gemachten Angaben.

28 Robert Leeson (1979) Travelling Brothers: The Six Centuries<Road from Craft Fellowship to Trade Unionism, London: Allen \& Unwin, 30. 
Unabhängigkeit... innerhalb eines Systems von Bräuchen und Gesetzen, die den Wettbewerb verhinderten und die Solidarität unter den Unternehmern des gleichen Handwerks förderte $<{ }^{29}$

Untermauert wurden die Zunftregeln vom »Statute of Artificers « von 1563, dem Handwerkergesetz, in dem in großem Umfang Gesetze, die zum Teil bis auf das 14. Jahrhundert zurückgeführt werden konnten, sowie lokale Geschäftspraktiken, die seit noch längerer Zeit Anwendung fanden, kodifiziert wurden. In Folge dieser Rechtsvorschriften waren die sich entwickelnden kapitalistischen Formen industrieller Organisation, die auf dem vertikal integrierten Unternehmen basierten, praktisch rechtswidrig. Kein Arbeitgeber konnte in einem Handwerkszweig tätig werden, in dem er keine Lehre absolviert hatte. Man konnte das Gesetz auch so verstehen, dass der Arbeitgeber daran gehindert werden sollte, Arbeiter aus verschiedenen Handwerksbranchen einzustellen und diese gemeinsam arbeiten zu lassen, weil er in diesem Fall neben seinem eigenen Handwerk noch eine Reihe anderer Handwerke ausgeübt hätte. ${ }^{30}$

Das umfangreiche Gesetzeswerk von 1563 verschwand jedoch nicht plötzlich. In England gab es kein kategorisches Verbot der Zünfte, wie in der entsprechenden Gesetzgebung der Französischen Revolution, dem »Decret Allarde« und der »Loi Le Chapelier«. In England verlief der Wandel eher allmählich und mehr als ein Jahrhundert lang stark umstritten. So war das Gesetz von $1814^{31}$, das die Ausbildungsbestimmungen des »Statute of Artificers « endlich abschaffte, eine eher symbolische Veränderung. Dieses Gesetz wurde dabei gerade deshalb verabschiedet, weil Gruppierungen in den städtischen Zünften gemeinsam den energischen Versuch unternommen hatten, das Gesetz von 1563 beizubehalten. Im Rahmen dieser Kampagne kam es zu breit angelegten Streikaktionen, Parlamentseingaben und Gerichtsprozessen, die alle das Ziel hatten, den Bestimmungen des Gesetzes Geltung zu verschaffen. ${ }^{32}$

Der Grund für die Abschaffung der Ausbildungsbestimmungen im Jahre 1814 war die Wirtschaftspolitik der damaligen Zeit, die für einen ungehinderten Wettbewerb eintrat. Joseph Chittys Lehrbuch über die Berufsausbildung, das 1812 als Antwort auf die auf Grund des Gesetzes von 1563 >zahlreichen vor kurzem eingeleiteten Gerichtsverfahren < erschienen war, veranlasste Adam Smith, T.R. Malthus und William Paley, sich für die Abschaffung des Gesetzes einzusetzen. Die Anstrengungen, das Gesetz durchzusetzen > wurden übereinstimmend unternommen, ungeachtet der Vorteile, die der Öffentlichkeit dadurch möglicherweise entstehen könnten, sondern ausschließlich zu Gunsten der Gesellen, um die Löhne hoch zu halten <. Die Abschaffung der Bestimmungen würde bewirken, dass sich >Wettbewerb mit freier Beschäftigung verbindet< und, wie Adam Smith plädierte, alle einen Nutzen davon hätten:

29 John Smail (1987) »New languages for labour and capital: the transformation of discourse in the early years of the industrial revolution«, 12 Social History, 54-61, 55.

30 Siehe Re. Statute of 5 Eliz., Apprentices (1591) 4 Leon 9, und die Diskussion dieses Fallrechts von Lord Mansfield CJ in Raynard v. Chase (1756) 1 Burr. 6.

3154 Geo. III, c. 96.

32 Siehe ganz allgemein Iorwerth Prothero (1979) Artisans and Politics in Early Nineteenth Century London: John Gast and His Times, Folkestone: Dawson. 
>Wo freier Wettbewerb herrscht, wird jeder Einzelne seine Arbeitskraft und sein Kapital immer dorthin leiten, wo sie seinem ureigenen Interesse am besten dienen; jeder weiß auf Grund einer Vielzahl an Erfahrungen für sich selbst am besten, was in seinem Interesse liegt; das Interesse der Allgemeinheit muss jedoch im allgemeinen sehr gut abgesichert werden, wenn das Interesse jeden einzelnen Individuums bestmöglich berücksichtigt wird ${ }^{33}$

Lord Kenyon hatte bereits früher behauptet, dass die >praktische Vernunft< des Marktes und nicht die von den Zünften ausgeübte Kontrolle das Problem der schlechten Warenqualität in den Griff bekommen werde: >[das Gesetz] wurde aus dem Grund gemacht, dass keine schlechten Waren in Umlauf gebracht werden sollten; doch die praktische Vernunft sagt uns, dass bei einem schlechten Produkt nicht die Gefahr besteht, dass es positiv von der Welt aufgenommen wird oder dem Zweck des Händlers dient.$^{34}$

Die Umwälzungen, die der Untergang der Zünfte mit sich brachte, wirkten sich möglicherweise langfristig auf die britische Industrialisierung aus. Auf dem europäischen Kontinent wiederholten die post-revolutionären Gesetzbücher die Verurteilung der >Korporationen des Ancien Régime durch die »Loi Le Chapelier« von 1791. Doch trotz dieses umfassenden gesetzlichen Verbots wurden einige Aspekte der Zünfte von den jetzt neu entstehenden Organisationsformen übernommen. Die Kombination von Wettbewerb und Kooperation sowie die Beibehaltung solidarischer Verbindungen der unabhängigen Produzenten untereinander charakterisierten die $>$ Industriedistrikte< in Italien, sowie Frankreich, Deutschland und Japan, die Wirtschaftswissenschaftler in den letzten Jahrzehnten des 20. Jahrhunderts wieder entdeckten. ${ }^{35}$ In Großbritannien hielten sich organisatorische Verbindungen über unabhängige Produktionseinheiten länger als auf dem europäischen Kontinent. Die Ursache dafür waren zum einen wohl die Werte des »Common Law«, die >Handelsbeschränkungen< kritisch gegenüber standen, zum anderen waren sie aber auch das Vermächtnis eines bestimmten industriellen Entwicklungsmusters. Obwohl in Großbritannien zunftähnliche Beziehungsgeflechte in dem auf dem Handwerk gründenden Gewerkschaftswesen, dessen Mittelpunkt der gewerkschaftspflichtige Betrieb bildete, fortbestanden, war der Kollektivismus auf der Arbeitgeberseite schwach ausgebildet und rückständig. Industriezusammenballungen und ein wachsender Trend hin zur vertikalen Integration bestimmten die Entwicklung im 20. Jahrhundert. ${ }^{36}$

In letzter Zeit hat sich dieser Trend umgekehrt. Sollte man in der vertikalen Desintegration der Produktion und der zunehmenden Verbreitung wirtschaftlicher Netzwerkformen eine Wiederkehr der Zünfte sehen?

33 Prothero (Fn. 32) 2.

34 Smith v. Company of Armourers (1799) Peake 199, 201.

35 Siehe ganz allgemein Michael Piore und Charles Sabel (1984) The Second Industrial Divide: Possibilities for Prosperity, New York: Basic Books.

36 Siehe Deakin und Wilkinson (Fn. 25) Kapitel 2 (über die vertikale Integration und den Aufstieg des Beschäftigungsmodells) und Kapitel 4 (über die Beibehaltung der zunftähnlichen Wirtschaftskontrolle im modernen britischen Gewerkschaftswesen). 
Verträge in der heutigen >Netzwerkwirtschaft $:$ die vertikale Desintegration im Rundfunk- und Fernsehsektor

Gemäß der einflussreichen These von Manuel Castells ${ }^{37}$, bewirkte die Kombination von technologischen und organisatorischen Wandlungen zum Ende des 20. Jahrhunderts eine Neuordnung des Kapitalismus, die zur Entstehung der >Netzwerk-Gesellschaft< führte. In Castells' Modell durchdringt das >Netzwerk< sowohl den Markt als auch das Unternehmen, indem es das (mittlerweile) traditionelle vertikal integrierte Unternehmen durch Organisationseinheiten ersetzt, die weniger eng miteinander verbunden sind, und teilt die Massenkonsummärkte in spezielle Segmente auf. Die Produktmarktderegulierung, die Liberalisierung der Handelsbeziehungen und die wachsende Bedeutung des Kapitalmarktes bei der Umstrukturierung von Unternehmen spielen eine wichtige Rolle beim Übergang in das globale Wirtschaftssystem. In diesem Zusammenhang ist jedoch vor allem die Technik die Triebfeder für den sozialen und wirtschaftlichen Wandel, wie bereits (gemäß Castells) während der ersten industriellen Revolution. Die >netzwerkbildende< Logik der neuen Informationstechnologien und Biowissenschaften findet sich in den flexiblen Sozialstrukturen der $>$ New Economy< wieder: >der »spirit of informationalism « ist die Kultur der »kreativen Zerstörung «, beschleunigt auf die Geschwindigkeit der optoelektronischen Kreisläufe, die die Signale verarbeiten $<$. Schumpeter trifft Weber im Cyberspace des >Netzwerk-Unternehmens $<.^{38}$ In diesem neuen Rahmen ist weder das Individuum, noch das Kollektiv, Unternehmen oder Staat die grundlegende Wirtschaftseinheit, sondern das Netzwerk selbst, >das aus einer Vielzahl von Individuen und Organisationen besteht und sich ständig verändert, da Netzwerke auf ein günstiges Umfeld und favorisierende Marktstrukturen reagieren $<.{ }^{39}$

Die Schlüsselfrage bei der Beurteilung von Castells These ist, ob die Trends, die er beschreibt, insgesamt ein wirklich neues Phänomen ergeben, oder ob sie lediglich eine weitere Phase in der bekannten Dynamik des industriellen Kapitalismus mit seiner Verbindung von Unternehmen und Markt darstellen. Im Sinne von Commons >MikroInstitutionalismus< könnte eine Fallstudie zur industriellen Entwicklung eines speziellen Wirtschaftssektors hilfreich sein. Die Kulturindustrie und besonders die Fernsehproduktion eignen sich dafür hervorragend, da man in diesen Bereichen alle Elemente der >New Economy< wieder findet: die herausragende Rolle der Informationstechnologie (vor allem durch die Einführung der digitalen Rundfunk- und Fernsehübertragung), die vertikale Desintegration bestehender Unternehmen und die scheinbare Neuordnung wirtschaftlicher Beziehungen in Form von anpassungsfähigen Netzwerken. Vor allem findet man ein Element, das Castells in seinen Ausführungen zwar erwähnt, aber nicht besonders hervorhebt: die Rolle des Wettbewerbsrechts als Katalysator für den wirtschaftlichen Wandel.

37 Castells (Fn. 3).

38 Castells (Fn. 3) 199.

39 Castells (Fn. 3) 198. 
Der Rundfunk- und Fernsehsektor im Vereinigten Königreich ist besonders deshalb interessant, weil es in diesem Bereich zwei parallele Ansätze gibt, Netzwerk-Beziehungen an Stelle von vertikal integrierten Organisationsstrukturen zu fördern: Zum einen bietet die Regierung Anreize für die Entwicklung eines unabhängigen Produktionssektors, der durch eine Quotenregelung bei der Vergabe von Subaufträgen und spezielle Vertragsbedingungen unterstützt wird. Zum anderen gibt es innerhalb der BBC einen >internen Markt<, der Vertragsbeziehungen im Rahmen eines organisatorischen Systems $>$ nachahmt $<{ }^{40}$

Letztlich bildete die Struktur der BBC vor den Reformen den Hintergrund für das Florieren einer modernen zunftähnlichen Kultur. Die ausgedehnten Studien über die BBC, die der Soziologe Tom Burn in den 60er und 70er Jahren des 20. Jahrhunderts durchführte, machten deutlich, bis zu welchem Grad die Mitarbeiter der BBC in diesem Zeitraum bereit waren, >sich selbst - und ganz bewusst - individuellen Zielen und Werten zu widmen, die mit den Zielen und Werten der öffentlich-rechtliche Sendeanstalten in Einklang standen, ohne dass sie dort unbedingt ihren Ursprung hatten $<$, und auf diese Art, wie es ein Personalchef dieser früheren Zeit nannte, >ein Wachstum schufen, das nichts kostete $<.{ }^{41}$ Was dies letztendlich bedeutete, kann man dem folgenden Kommentar eines Arbeitnehmervertreters über die Reformen der 90er Jahre entnehmen: >als die Quotenregelung für unabhängige Unternehmen ins Spiel kam und man damit begann, nach und nach Reinigungsdienst, Catering und Sicherheitsdienst auszulagern, kämpften die meisten Angestellten darum, ihre Jobs zu behalten, sie sagten keineswegs >Was für eine Chance! <. Dies führte dazu, dass >es so war, als ob man für irgendeinen Sender arbeitete, es war den Angestellten egal, dass es die BBC war $<{ }^{42}$

Andererseits sahen ihre Befürworter die Umstrukturierung der BBC unter dem Aspekt, dass man von einem vom Produzenten geleiteten, streng bürokratischen Produktionsprozess abrückte, der als kreativitätshemmend galt. Die Einführung von marktähnlichen Prozessen und flexiblen Organisationsformen würde, so hoffte man, kreative Fähigkeiten freisetzen und die Innovation fördern. Doch statt dessen entstand ein >Scheinmarkt $<$ mit einzelnen Produktions- und Auftragsstufen, im Falle der BBC innerhalb organisatorischer Grenzen, im Falle der Auslagerung an unabhängige Unternehmen außerhalb dieser Grenzen. In den späten 90er Jahren wurde der interne Markt der BBC durch Produktionsgarantien stabilisiert, die die innerbetrieblichen

40 Dieser Abschnitt basiert auf Ideen, die gemeinsam mit Steve Pratten und Ana Lourenço entwickelt wurden; siehe Simon Deakin und Stephen Pratten (1999) »Reinventing the market? Competition and regulatory change in broadcasting «, 26 Journal of Law and Society, 323-350 und Simon Deakin, Ana Lourenço und Stephen Pratten (2006) »No third way for economic organisation? Networks and quasi-markets in broadcasting «, Working Paper, Centre for Business Research, University of Cambridge, zu weiteren Informationen über den institutionellen Hintergrund der vertikalen Desintegration im Rundfunk- und Fernsehsektor.

41 Tom Burns (1994) Description, Explanation and Understanding, Edinburgh: Edinburgh University Press, 91.

42 Zitate aus Interviewaufzeichnungen, Deakin, Lourenço und Pratten (Fn. 40). 
Subunternehmer schützten, während sie die Möglichkeiten der Einflussnahme seitens der unabhängigen Unternehmer beschränkten. ${ }^{43}$ Doch in letzter Zeit, vielleicht auch auf Grund der Tatsache, dass die Regierung eine Reform der BBC durchsetzen will, legt man wieder mehr Wert auf die Auslagerung von Produktionsfunktionen. Der Anteil der an den unabhängigen Sektor vergebenen Produktionen stieg dadurch weiter an, ${ }^{44}$ während der Anteil an innerbetrieblichen Produktionen erneut zurückging.

Gleichzeitig rückte man innerhalb des unabhängigen Sektors zu Gunsten einer Neuzuteilung von Eigentumsrechten gemäß den Vertragsbestimmungen zwischen Sender und Subunternehmer vom garantierten Subunternehmervertrag ab. So sollte sichergestellt werden, dass geistige Eigentumsrechte an der Wiederholung von Fernseh-Programmen auf die Programmmacher und nicht auf die Sendeanstalten übergehen. Einem kleinen Segment des unabhängigen Sektors wurde es möglich, sich dadurch neben den Senderechten für seine Programme auch eine stille Reserve in Form von sekundären und tertiären Verkaufsrechten zu sichern. Das hatte wiederum zur Folge, dass die Fremdfinanzierung durch Risikokapital und Private Equity in diesem Wirtschaftszweig anstieg. ${ }^{45}$

Nach einem Bericht der Medienaufsicht »Independent Television Commission (ITC)《 von Anfang 2000 über den unabhängigen Sektor, >ist ein gesunder und wettbewerbsfähiger Markt für Fernsehprogramme ein wichtiger Teil unserer kreativen Wirtschaft $<.{ }^{46}$ Dafür braucht man dann wieder einen unabhängigen Sektor, der >für sich allein lebensfähig und nicht auf eine Quotenregelung angewiesen ist $<.{ }^{47}$ Der ITC Report stellte jedoch auch einen möglichen Qualitätsverlust bei den Produktionen fest, der seine Ursache in der Tendenz unabhängiger Produzenten hat, Projekte mit geringem Risiko und Standardformaten zu realisieren, um sich so kontinuierlich mit Aufträgen zu versorgen. Die ITC machte den Vorschlag, das Problem zu lösen, indem man den Wettbewerb weiter verschärft: eine Eindämmung des festgestellten Missbrauchs der marktbeherrschenden Stellung der BBC, Vertragsbedingungen, die früher von privaten Fernsehunternehmen angewendet wurden und der Versuch, die Auftragsvergabe zu vereinfachen, würden den unabhängigen Sektor von den Kräften befreien, die seine Entwicklung blockierten. Wenn die altmodischen >Heimarbeit<-Unternehmen erst einmal an den Rand gedrängt wären, so erwartete man, würden die überlebenden Unter-

43 Deakin und Pratten (Fn. 40).

44 Das sogenannte $>$ Fenster des kreativen Wettbewerbs<, das 2004 angekündigt wurde und nach der Erneuerung der BBC Charter 2006 realisiert werden soll, sieht vor, dass neben den bereits garantierten 25\% weitere 25\% der Programmausgaben der BBC für den Wettbewerb zwischen BBC-eigenen Produzenten und dem unabhängigen Sektor zur Verfügung stehen. Bzgl. der Diskussion über die Auswirkungen einer größeren Beteiligung des unabhängigen Sektors seitens der BBC siehe Will Hutton, Áine O'Keefe und Natalie Turner (2005) The Tipping Point: How Much is Broadcast Creativity at Risk? An Independent Report commissioned by the $B B C$, London: The Work Foundation.

45 Deakin, Lourenço und Pratten (Fn. 40).

46 Independent Television Commission (2002) A Review of the UK Programme Supply Market, London: ITC, Abschnitt 9. Das ITC wurde 2003 von der derzeitigen britischen Medienaufsicht Ofcom übernommen.

47 Independent Television Commission (Fn. 46) Abschnitt 10. 
nehmen in der Lage sein, die Kontrolle über sekundäre und tertiäre Rechte zu erlangen, und hätten bessere Voraussetzungen Fremdkapital anzuziehen.

Doch in letzter Zeit wurden im Fernsehproduktionssektor noch weitere Erfahrungen gemacht. Das Modell der Mehrkosten-Finanzierung, das in Folge des »Broadcasting Act « von 1990 eingeführt wurde, erschwerte zwar einigen der kleineren unabhängigen Unternehmen das Wachstum, schützte sie aber zugleich vor dem Risiko überhöhter Produktionskosten. Mit diesem Problem haben zwar alle Fernsehproduktionen zu kämpfen, doch nur die größeren Anbieter können damit fertig werden, weil sie über die notwendigen Reserven verfügen. Man müsste wahrscheinlich die Übertragungsund Produktionsfunktionen der BBC formell entbündeln, um allen unabhängigen Unternehmen die gleichen Bedingungen bieten zu können. Doch, wie das ITC bereits erkennen musste, >könnte die strukturelle Trennung der Übertragungs- und Produktionsgeschäfte der BBC dazu führen, dass die BBC-eigenen Produzenten und die unabhängigen Unternehmen zwar die gleichen Chancen hätten, dies aber wahrscheinlich beträchtliche Kosten für das Unternehmen verursachen würde $<{ }^{48}$ Die Entwicklung der BBC seit den späten 90er Jahren, die einen beachtlichen Wandel des unternehmensinternen Marktes mit sich brachte, weist zudem auf die potentielle zerstörerische Wirkung einer Zersplitterung der Organisation auf die Produktionsfähigkeit hin. ${ }^{49}$

Wenn wir hier die Rolle netzwerkartiger Beziehungen erkennen, mit einem Vertragsgewebe, dessen Knotenpunkte die größten Sendeanstalten bilden, und einer Vielzahl von Organisationsformen, die schnell den Bedarf an abwechslungsreichen Qualitätsproduktionen decken können, dann sehen wir auch die möglichen Grenzen der Netzwerkbildung in einem stark deregulierten Umfeld. Der Vorstoß, diesen Sektor marktmäßig zu gestalten, führte sehr schnell zu Bedenken bezüglich der Produktionsqualität in einem Bereich, in dem sich schon die bestehenden Qualitätsabsprachen als wenig zuverlässig erweisen. Das Ergebnis der Reformen war die Unterminierung der >zunftähnlichen< Autonomie der Produzenten, die einstmals ein Garant für Qualität war.

\section{Schlussbemerkungen}

$\mathrm{Zu}$ Beginn der Debatte über neue Formen wirtschaftlicher Organisation in den 80er Jahren vertraten Michael Piore und Charles Sabel ${ }^{50}$ die Ansicht, dass die vertikale Integration der Produktion nicht historisch unvermeidlich war, sondern eher ein Zufallsprodukt in einer bestimmten Phase des industriellen Kapitalismus. Sie sagten das Wiederaufleben von Netzwerken voraus und wiesen so auf die mögliche Wiederkehr von Regulierungstypen hin, die dem ersten großen >industrial divide< im 19. Jahrhundert vorausgingen. Doch zwanzig Jahre später gibt es kaum Anzeichen dafür, dass es dazu

48 Independent Television Commission (Fn. 46) Abschnitt 31.

49 Siehe allgemein Georgina Born (2005) Uncertain Vision: Birt, Dyke and the Reinvention of the BBC, London: Vintage; Hutton, O'Keefe und Turner (Fn. 44).

50 Piore und Sabel (Fn. 35). 
kommen wird. Das beachtliche Versprechen, das die Netzwerke gaben, droht von einer Neo-Schumpeterischen Sicht der Wirtschaft und Gesellschaft verdrängt zu werden, in der technologischer Determinismus wenig oder gar keinen Platz lässt für eine institutionelle Vielfalt. Entscheidend ist die Wettbewerbspolitik. Sie muss die Vielfalt der Erscheinungsformen erkennen, in der sich Wettbewerb äußert und zudem verstehen, welche Regulierungsinstrumente geeignet sind, den Mechanismus in Gang zu setzen, der traditionell - trotz hierarchischer Kontrolle einerseits und der homogenisierenden Kraft der Massenmärkte andererseits - die Autonomie der Hersteller förderte. Dann könnte es auch zu einer Wiederkehr der Zünfte kommen. 